\title{
|||||||||||||||||||||||||||||||||||||||||||||||||||||||||||||||||||.
}

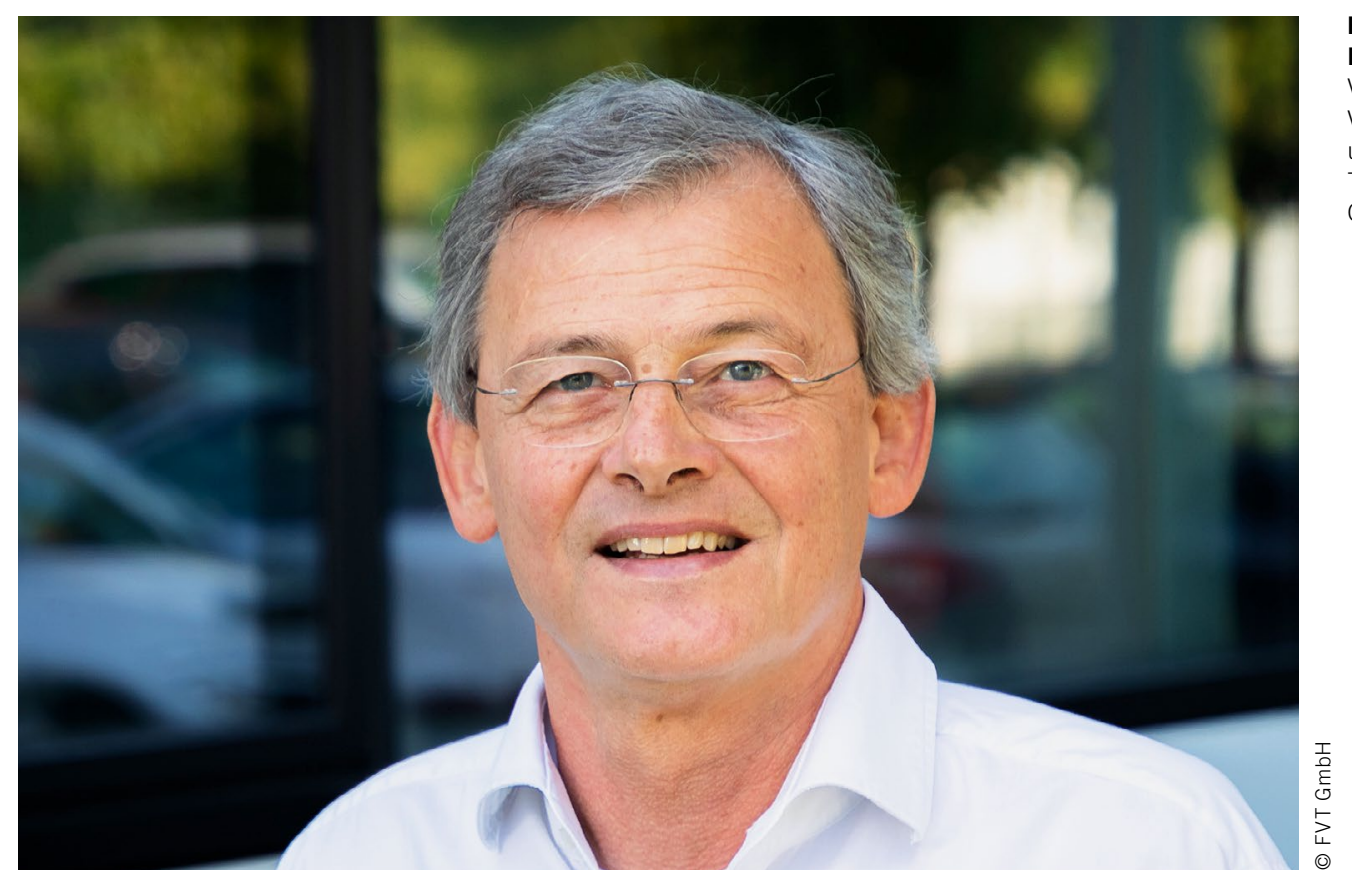

Prof. Dipl.-Ing. Dr. Helmut Eichlseder Vorstand des Instituts für Verbrennungskraftmaschinen und Thermodynamik der Technische Universität Graz (Österreich)

\section{Abschalteinrichtungen in Kraftfahrzeugen}

Viele Klagen beschäftigten und beschäftigen sich derzeit mit der Fragestellung von sogenannten Abschalteinrichtungen von emissionsmindernden Maßnahmen im Pkw. Im Zusammenhang mit dem Dieselskandal aufgekommen, wurde diese vorerst allgemein auf die Abschaltung oder Reduktion emissionsmindernder Maßnahmen wie der Abgasrückführung oder SCRReduktionsmitteldosierung bezogen und nicht spezifisch auf eine emissionsmindernde Technologie gemünzt. Gemeint war zunächst vor allem die aufgrund der Erkennung einer stattfindenden Emissionsmessung (Zykluserkennung) reduzierte Nutzung oder Abschaltung dieser Technologien, sodass im Betrieb auf der Straße deutlich höhere Emissionen auftreten. Hier ist es vergleichsweise einfach, dies als unrechtmäßig - um nicht zu sagen betrügerisch - zu erkennen und zu qualifizieren. Die Unzulässigkeit einer solchen Abschalteinrichtung mit dem Sachverhalt einer Zykluserkennung ist außer Diskussion, was unter anderem auch das deutsche Kraftfahrt-Bundesamt (KBA) bereits 2015 festgestellt hat und hier nicht weiter betrachtet werden soll.

Darüber hinaus wurde in der jüngeren Zeit in mehreren Verfahren die Rechtmäßigkeit von temperaturabhängigen Emissionsminderungsmaßnahmen wie der Abgasrückführung in Abhängigkeit von der Umgebungstemperatur, bezeichnet als sogenanntes Thermofenster, geführt. Hier ist aus meiner Sicht, dem Blickwinkel eines Technikers ohne qualifizierte juristische Bildung, die Sache nicht so einfach zu beurteilen. Ohne eine Beschönigung oder Verniedlichung vorzunehmen: Dass durch thermodynamisch bedingte Effekte wie Kondensationsvorgänge,
Stoffeigenschaften ganz allgemein sowie thermomechanische Beanspruchungen etc. Einschränkungen und Grenzen bestehen, liegt in der Natur technischer Einrichtungen.

Auch im gesamten möglichen Temperaturbereich des Fahrzeugbetriebs von -25 bis $+40^{\circ} \mathrm{C}$ sowie höhen- (und damit Luftdruck-) unabhängig wird kein absolut gleiches Start-, Ansprech- und Volllastverhalten zu erwarten sein. Bei Elektrofahrzeugen ergeben sich damit beispielsweise beträchtliche Unterschiede in der Reichweite.

Es ist zu hoffen, dass bei der juristischen Bewertung der Funktionalität der Emissionsminderungsmaßnahmen die Einhaltung der Luftqualität und der reale Sinn für die Umwelt im Vordergrund stehen. Wie bei einer statistischen Toleranzrechnung sollte hier nicht die spitzfindig definierte theoretisch maximal mögliche Kombination aller negativen Parameter die (damit kaum vernünftig machbare) Auslegung bestimmen, sondern die Relevanz für die Luftqualität. Mit der Einführung der RDE-Gesetzgebung ist hier ein Schritt gelungen, der zu Recht als signifikant bezeichnet wird und der bereits heute repräsentative Vorgaben hinsichtlich der Umgebungsbedingungen enthält. Im Gegensatz zu einer Auslegung, die singulär auf Extrembedingungen zugespitzt ist und unrealistischen Ressourcenaufwand bedingt, kann mit einer luftqualitätsorientierten Auslegung rasch in die Breite gegangen und damit Wirkung entfaltet werden. Daher ist zu hoffen, dass diese faktenbasierte Bewertung auch Niederschlag in die folgenden juristischen Verfahren findet. 